\title{
Multi-wavelength afterglow observations of the high redshift GRB 050730
}

\author{
S. B. Pandey ${ }^{1}$, A. J. Castro-Tirado ${ }^{1}$, S. McBreen ${ }^{2,3}$, M. D. Pérez-Ramírez ${ }^{4}$, M. Bremer ${ }^{5}$, M. A. Guerrero ${ }^{1}$, A. Sota ${ }^{6}$,
} B. E. Cobb ${ }^{7}$, M. Jelínek ${ }^{1}$, A. de Ugarte Postigo ${ }^{1}$, J. Gorosabel ${ }^{1}$, S. Guziy ${ }^{1,15}$, C. Guidorzi ${ }^{9}$, C. D. Bailyn ${ }^{7}$, T. Muñoz-Darias ${ }^{8}$, A. Gomboc ${ }^{9,10}$, A. Monfardini ${ }^{9}$, C. G. Mundell ${ }^{9}$, N. Tanvir ${ }^{11}$, A. J. Levan ${ }^{11}$, B. C. Bhatt ${ }^{12,13}$, D. K. Sahu ${ }^{12,13}$, S. Sharma ${ }^{14}$, O. Bogdanov ${ }^{15}$, and J. A. Combi ${ }^{4}$

1 Instituto de Astrofísica de Andalucía, PO Box 03004, 18080 Granada, Spain e-mail: sbp2@mssl.ucl.ac.uk

2 European Space Agency, ESTEC, Keplerlaan 1, 2201 AZ Noordwijk, The Netherlands

3 Max-Planck-Institut für extraterrestrische Physik, 85748 Garching, Germany

${ }^{4}$ Departamento de Física (EPS), Universidad de Jaén, Campus Las Lagunillas s/n (Ed-A3), 23071 Jaén, Spain

5 Institut de Radioastronomie Millimétrique, 300 rue de la Piscine, 38406 Saint Martin d'Hères, France

${ }^{6}$ IAA-CSIC and Space Telescope Science Institute, St. Martin Dr., Baltimore, MA, USA

7 Department of Astronomy, Yale University, PO Box 208101, New Haven, CT 06520, USA

${ }^{8}$ Instituto de Astrofísica de Canarias, C/. Vía Láctea, s/n, 38200 La Laguna, Tenerife, Spain

9 Astrophysics Research Institute, Liverpool John Moores University, Twelve Quays House, Egerton Wharf, Birkenhead, CH41 1LD, UK

10 Faculty of Mathematics and Physics, University of Ljubljana, Jadranska 19, 1000 Ljubljana, Slovenia

11 Center for Astrophysics Research, University of Hertfordshire, College Lane, Hatfield AL10 9AB, UK

12 Center for Research \& Education in Science \& Technology, Hosakote, Bangalore 562 114, India

13 Indian Institute of Astrophysics, Bangalore 560 034, India

14 Aryabhatta Research Institute of Observational Sciences (ARIES), Manora Peak, Naini Tal 263129, Uttaranchal, India

15 Nikolaev State University, Nikolska 24, Nikolaev 54030, Ukraine

Received 11 February 2006 / Accepted 2 August 2006

\section{ABSTRACT}

\begin{abstract}
Context. GRB 050730 is a long duration high-redshift burst $(z=3.967)$ that was discovered by Swift. The afterglow shows variability and was well monitored over a wide wavelength range. We present comprehensive temporal and spectral analysis of the afterglow of GRB 050730 including observations covering the wavelength range from the millimeter to X-rays.

Aims. We use multi-wavelength afterglow data to understand the complex temporal and spectral decay properties of this high redshift burst.

Methods. Five telescopes were used to study the decaying afterglow of GRB 050730 in the $B, V, r^{\prime}, R, i^{\prime}, I, J$ and $K$ photometric pass bands. A spectral energy distribution was constructed at $2.9 \mathrm{~h}$ post-burst in the $B, V, R, I, J$ and $K$ bands. X-ray data from the satellites Swift and XMM-Newton were used to study the afterglow evolution at higher energies.

Results. The early afterglow shows variability at early times and the slope steepens at 0.1 days (8.6 ks) in the $B, V, r^{\prime}, R, i^{\prime}, I, J$ and $K$ passbands. The early afterglow light curve decayed with a powerlaw slope index $\alpha_{1}=-0.60 \pm 0.07$ and subsequently steepened to $\alpha_{2}=-1.71 \pm 0.06$ based on the $R$ and $I$ band data. A millimeter detection of the afterglow around 3 days after the burst shows an excess in comparison to theoretical predictions. The early X-ray light curve observed by Swift is complex and contains flares. At late times the X-ray light curve can be fit by a powerlaw decay with $\alpha_{x}=-2.5 \pm 0.15$ which is steeper than the optical light curve. A spectral energy distribution (SED) was constructed at $\sim 2.9 \mathrm{~h}$ after the burst. An electron energy index, $p$, of $\sim 2.3$ was calculated using the SED and the photon index from the X-ray afterglow spectra and implies that the synchrotron cooling frequency $v_{\mathrm{c}}$ is above the X-ray band.
\end{abstract}

Key words. gamma ray: bursts - techniques: photometric

\section{Introduction}

GRB 050730 is one of a growing number of known gamma-ray bursts (GRBs) at high redshift for which have peculiar superimposed variability from optical to X-rays (e.g. GRB050904: Böer et al. 2005; Cusumano et al. 2006; Watson et al. 2005). The observed variability contains a wealth of information about the nature of the burst and its afterglow (Burrows et al. 2005). The very early X-ray and optical observations of GRB 050730 were possible due to the very fast slew time of $S$ wift satellite (Gehrels et al. 2004).

The long-duration burst GRB 050730, was detected by the BAT instrument on Swift (trigger $=148225)$ at $T_{0}=19^{\mathrm{h}} 58^{\mathrm{m}} 23^{\mathrm{s}}$ UT on 30th July 2005 (Holland et al. 2005). The X-ray and the optical afterglow of the burst were discovered by the onboard instruments XRT and UVOT respectively, after $132 \mathrm{~s}$ and $119 \mathrm{~s}$ after the BAT trigger (Holland et al. 2005). The optical afterglow (OA) candidate was later confirmed by ground based 
Table 1. PdBI dual frequency observations of GRB 050730. The first three rows refer to the $3 \mathrm{~mm}$ band and the last 3 rows refer to the $1 \mathrm{~mm}$ band. The flux errors are $1 \sigma$. The last two columns list the beam size and position angle (PA) respectively.

\begin{tabular}{cccccl}
\hline \hline Start time & End time & Freq. [GHz] & Flux [mJy] & Beam( $\left(^{\prime \prime} \times{ }^{\prime \prime}\right)$ & PA $\left(^{\circ}\right)$ \\
\hline 2005 Aug. 2.659 & 2.816 & 102.746 & $2.74 \pm 0.94$ & $9.2 \times 5.7$ & 49 \\
2005 Aug. 5.577 & 5.838 & 105.304 & $1.34 \pm 0.51$ & $6.9 \times 6.4$ & 73 \\
2006 Jan. 5.193 & 5.356 & 86.847 & $-0.24 \pm 0.27$ & $4.4 \times 2.9$ & 4 \\
\hline 2005 Aug. 2.659 & 2.816 & 213.233 & $4.39 \pm 4.21$ & $4.8 \times 2.5$ & 10 \\
2005 Aug. 5.577 & 5.838 & 214.977 & $-5.13 \pm 3.81$ & $3.8 \times 2.9$ & 0 \\
2006 Jan. 5.193 & 5.356 & 230.538 & $-4.21 \pm 1.93$ & $1.7 \times 1.1$ & 1 \\
\hline
\end{tabular}

observations using the Sierra Nevada $1.5 \mathrm{~m}$ telescope by Sota et al. (2005). The near infra-red (NIR) afterglow was discovered by Cobb \& Bailyn (2005) using ANDICAM on CTIO $1.3 \mathrm{~m}$. Spectroscopic observations of the afterglow candidate, obtained $\sim 4 \mathrm{~h}$ after the burst, using the MIKE echelle spectrograph on Magellan II, derive a redshift of $z=3.967$ (Chen et al. 2005a). The value of redshift value was further confirmed by Rol et al. (2005) and Prochaska et al. (2005). The measured redshift was based on the strong absorption feature at $\lambda 6040 \AA$ that was identified with hydrogen Ly- $\alpha$ and other narrow absorption lines from heavy ions originating in the vacinity of the GRB progenitor (Chen et al. 2005a,b).

The source was initially detected by Swift/BAT at $\operatorname{RA}(J 2000)=14^{\mathrm{h}} 08^{\mathrm{m}} 16.40^{\mathrm{s}}, \operatorname{Dec}(\mathrm{J} 2000)=-03^{\circ} 45^{\prime} 41^{\prime \prime}$. 1 with an uncertainty of $3^{\prime}$. Markwardt et al. (2005) reported the burst duration $\left(T_{90}\right)$ of $155 \pm 20 \mathrm{~s}$ and a fluence of $(4.4 \pm 0.4) \times$ $10^{-6} \mathrm{erg} \mathrm{cm}^{-2} \mathrm{~s}^{-1}$. Initial analysis of XRT data (130 to $1000 \mathrm{sec}-$ onds after the burst) show flaring in the light-curve (Grupe et al. 2005; Perri et al. 2005; Starling et al. 2005). The $S$ wift/XRT error box was also observed by XMM-Newton (Schartel 2005) and confirmed the presence of the afterglow of GRB 050730 (Ehle \& Juarez 2005). VLA radio observations around 3 days after the burst show a weak radio source consistent with the optical afterglow candidate at $8.5 \mathrm{GHz}$ (Cameron 2005). Three WSRT observations at $4.9 \mathrm{GHz}$ (van der Horst \& Rol 2005a,b) at 4.6, 6.6 and 12.6 days postburst found no significant flux at the radio afterglow position.

This paper is organized as follows. Afterglow observations spanning a wide wavelength range, including millimeter, nearinfrared (NIR), optical and X-rays, are described in Sect. 2. The results of the multi-wavelength analysis are presented in Sect. 3. A detailed discussion of the afterglow light-curves, spectral energy distribution and their comparison to model predictions can be found in Sect. 4. Finally, concluding remarks are presented in Sect. 5 .

\section{Afterglow observations of GRB 050730}

In the following sections we describe the observations, data reduction and calibration of the afterglow of GRB 050730 at millimeter, NIR, optical and X-ray wavelengths.

\subsection{Millimeter wave observations}

Observations were triggered at the Plateau de Bure Interferometer (PdBI, Guilloteau et al. 1992) as part of an on-going Target of Opportunity (ToO) programme. The observations were centered on the equatorial coordinates $\operatorname{RA}(J 2000)=14^{\mathrm{h}} 08^{\mathrm{m}} 17.14^{\mathrm{s}}, \quad \operatorname{Dec}(\mathrm{J} 2000)=-03^{\circ} 46^{\prime} 17^{\prime \prime} .8$. The counterpart was observed on August 2 and 5, 2005 in a compact 5 antenna configuration (5D) and on January 5, 2006 in a 6 antenna configuration (6Cp). The later observation in January was designed to assess the influence of the underlying host galaxy on the initial afterglow measurements. Standard software packages, CLIC and MAPPING, distributed by the Grenoble GILDAS group ${ }^{1}$ were used to reduce the data. Flux calibration was based on the carbon-star MWC349. The flux values and error estimates were established with point source fits in the UV plane, which were fixed to the phase center coordinates.

The afterglow continuum was tentatively detected in the $3 \mathrm{~mm}$ band on August 2 and 5 at 2.9 and $2.6 \sigma$ respectively (Table 1). A combination of both data sets (with the appropriate weighting for system temperature and amplitude calibration) gave a flux of $1.73 \pm 0.45 \mathrm{mJy}$, i.e. a $3.8 \sigma$ detection on the phase center. Only upper limits could be obtained in the $1 \mathrm{~mm}$ band.

A final observation on January 5th, 2006 under good atmospheric conditions provided upper limits in both the $3 \mathrm{~mm}$ and $1 \mathrm{~mm}$ bands, indicating that the contribution of the host galaxy is not significant.

\subsection{Near-IR observations}

The NIR observations of the afterglow started at 22:46 UT on 30th July 2005 under non-photometric sky conditions using the ANDICAM instrument mounted on the $1.3 \mathrm{~m}$ telescope at Cerro Tololo Inter-American Observatory $(\mathrm{CTIO})^{2}$ and operated as a part of the Small and Moderate Aperture Research Telescope System (SMARTS) consortium ${ }^{3}$. The ANDICAM detector consists of a dual-channel camera that allows simultaneous optical $(V, I)$ and NIR $(J, K)$ imaging. NIR and optical images are acquired simultaneously by the ANDICAM instrument via an internal mirror which repositions the NIR image on the CCD, essentially "dithering" without physically moving the telescope and interrupting the optical observations.

A combination of telescope pointings and internal dithers were used to obtain $16 \mathrm{~J}$ and $16 \mathrm{~K}$ band images. The reduction process was as follows. A master dome flat was produced for each of the 4 internal dither positions and the images were divided by the relevant master flat field. A sky frame in the $J$ and $K$ band for each dither position was obtained by the median combination of 4 images. Sky frames were subtracted from each image with rescaling to compensate for changes in brightness. The field was re-observed on subsequent photometric nights for calibration purposes. Standard fields Persson-P9104 and LCOBRI0021 (Persson et al. 1998) were used for NIR calibrations. The 16 reduced $J$ and $K$ band images were combined into sets of 4 or 8 to search for variability of the afterglow. The log of the $J$ and $K$ band observations on performed on the 30 and 31 July 2005 are listed in Table 2.

\footnotetext{
${ }^{1}$ GILDAS is a software package distributed by the IRAM Grenoble GILDAS group.

${ }^{2}$ http://WWW.astronomy.ohio-state.edu/ANDICAM

${ }^{3}$ http://www. astro.yale.edu/smarts
} 
$I$ and $V$-band images were also obtained by ANDICAM on 30 July and 1 August 2005. Standard reduction was performed on the optical images, including over-scan bias subtraction, zero subtraction and flat fielding as discussed in the following subsection. The log of these observations is also presented in Table 2.

\subsection{Optical observations}

Broad-band observations of the optical afterglow in the Bessel $B, V, R, I$ bands were carried out at various epochs between 30 July to 01 August 2005 using the 2-m Himalayan Chandra Telescope (HCT) of the Indian Astronomical Observatory (IAO, Hanle India), the $1.5 \mathrm{~m}$ telescope at Observatorio de Sierra Nevada (OSN) in Granada (Spain), the $1.3 \mathrm{~m}$ CTIO on Kitt Peak Arizona USA and the Instituto de Astrofísica de Canarias (IAC) $0.8-\mathrm{m}$ telescope at Observatorio de Izaña in Tenerife, Spain. Bessel $B, V$ and SDSS $r^{\prime}, i^{\prime}$ observations of the OA were obtained on 30 July 2005 using the 2-m robotic Liverpool Telescope (LT) of John Moores University at Canary Islands (La Palma, Spain). Several twilight flat and bias frames were also obtained during the observing runs for CCD calibrations. In order to improve the signal-to-noise ratio of the OA the data have been binned in $2 \times 2$ pixel and images were co-added when necessary. The magnitudes were determined by profile fitting of the images using a standard procedure in DAOPHOT/IRAF ${ }^{4}$.

During good photometric sky conditions at the Naini Tal ${ }^{5}$ $1.04 \mathrm{~m}$ reflector, the CCD $B, V, R$, and $I$ observations of the OA field and the Landolt (1992) standard PG1323-085 region were obtained on 31 Dec./01 Jan. 2006 along with several twilight flat and bias frames on 31 December and 01 January 2006 for calibration purposes at similar airmass values. The values of atmospheric extinction coefficients in $B, V, R$ and $I$ filters were $0.27,0.17,0.11$ and 0.09 mag respectively. The observed standard stars in the PG1323-085 region cover a range of $-0.13<(V-I)<0.83$ in color and of $12.1<V<14.0$ in brightness. The zero points and the associated errors were determined using standard DAOPHOT/IRAF routines and nearby stars. The calibrated $B, V, R$ and $I$ magnitudes of 10 nearby stars to the afterglow candidate are tabulated in Table 3 . The $B, V, R, I$ magnitudes of the afterglow candidate are calibrated differentially with respect to these secondary standards (Table 3 ) and the magnitudes of the afterglow candidate derived in this way are given in Table 2.

\subsection{X-ray observations}

\subsubsection{Swift observations}

The XRT on Swift began to observe GRB 050730 (Trigger 148225) $132 \mathrm{~s}$ after the trigger and the data confirm the presence of a decaying X-ray source in the Swift/XRT field at position $\operatorname{RA}(\mathrm{J} 2000)=14^{\mathrm{h}} 08^{\mathrm{m}} 17.5^{\mathrm{s}}, \operatorname{Dec}(\mathrm{J} 2000)=-03^{\circ} 46^{\prime} 19^{\prime \prime} .0$ with an uncertainty of $6^{\prime \prime}$ as reported by Perri et al. (2005). The XRT data consist of Window Timing (WT) data after $T_{0}+132 \mathrm{~s}$ to $T_{0}+790 \mathrm{~s}$ and Photon Counting (PC) data from $T_{0}+4 \mathrm{ks}$ and onwards. The data were reduced using the standard pipeline for XRT data analysis software ${ }^{6}$ (version 2.2) using the most recent calibration files. The data were analyzed with the XSPEC version 11.3 (Arnaud 1996).

\footnotetext{
4 http://iraf.noao.edu/

5 http://aries.ernet.in/

6 http://swift.gsfc.nasa.gov/docs/software/lheasoft/ download.html
}

Table 2. Observational $\log$ of the photometric CCD magnitudes in Bessell $B, V, R, I$, SDSS $r^{\prime}, i^{\prime}$ broad-band optical and $J, K$ NIR observations of the GRB 050730 afterglow.

\begin{tabular}{|c|c|c|c|}
\hline $\begin{array}{c}\text { Date (UT) of } \\
2005\end{array}$ & Magnitude & $\begin{array}{l}\text { Exposure time } \\
\text { (s) }\end{array}$ & Telescope \\
\hline \multicolumn{4}{|c|}{$B-$ passband } \\
\hline July 30.8608 & $19.68 \pm 0.04$ & 300 & OSN $1.5 \mathrm{~m}$ \\
\hline July 30.8745 & $19.61 \pm 0.03$ & 300 & OSN $1.5 \mathrm{~m}$ \\
\hline July 30.8899 & $19.77 \pm 0.04$ & 300 & OSN $1.5 \mathrm{~m}$ \\
\hline July 30.9567 & $\begin{array}{l}19.96 \pm 0.18 \\
V-\text { passband }\end{array}$ & 240 & LT $2.0 \mathrm{~m}$ \\
\hline July 30.8572 & $18.00 \pm 0.01$ & 300 & OSN $1.5 \mathrm{~m}$ \\
\hline July 30.8718 & $18.21 \pm 0.01$ & 300 & OSN $1.5 \mathrm{~m}$ \\
\hline July 30.8863 & $18.28 \pm 0.02$ & 300 & OSN $1.5 \mathrm{~m}$ \\
\hline July 30.9018 & $18.60 \pm 0.02$ & 300 & OSN $1.5 \mathrm{~m}$ \\
\hline July 30.9162 & $18.69 \pm 0.04$ & 300 & OSN $1.5 \mathrm{~m}$ \\
\hline July 30.9528 & $18.56 \pm 0.08$ & 240 & LT $2.0 \mathrm{~m}$ \\
\hline July 30.9536 & $18.67 \pm 0.12$ & 225 & CTIO $1.3 \mathrm{~m}$ \\
\hline July 30.9604 & $18.92 \pm 0.08$ & 225 & CTIO $1.3 \mathrm{~m}$ \\
\hline July 30.9623 & $18.92 \pm 0.06$ & $225 \times 4$ & CTIO $1.3 \mathrm{~m}$ \\
\hline July 30.9638 & $18.92 \pm 0.07$ & 225 & CTIO $1.3 \mathrm{~m}$ \\
\hline July 30.9711 & $\begin{array}{l}19.13 \pm 0.07 \\
r^{\prime}-\text { passband }\end{array}$ & 225 & CTIO $1.3 \mathrm{~m}$ \\
\hline July 30.8625 & $16.78 \pm 0.20$ & 120 & $\mathrm{LT} 2.0 \mathrm{~m}$ \\
\hline July 30.8670 & $17.11 \pm 0.10$ & 240 & LT $2.0 \mathrm{~m}$ \\
\hline July 30.8747 & $17.29 \pm 0.05$ & 360 & LT $2.0 \mathrm{~m}$ \\
\hline July 30.8841 & $17.46 \pm 0.04$ & 240 & LT $2.0 \mathrm{~m}$ \\
\hline July 30.8872 & $17.50 \pm 0.04$ & 240 & LT $2.0 \mathrm{~m}$ \\
\hline July 30.8991 & $17.74 \pm 0.04$ & 240 & LT $2.0 \mathrm{~m}$ \\
\hline July 30.9022 & $17.84 \pm 0.05$ & 240 & LT $2.0 \mathrm{~m}$ \\
\hline July 30.9426 & $17.70 \pm 0.05$ & 240 & LT $2.0 \mathrm{~m}$ \\
\hline July 30.9604 & $\begin{array}{l}17.88 \pm 0.04 \\
R-\text { passband }\end{array}$ & 240 & LT $2.0 \mathrm{~m}$ \\
\hline July 30.8536 & $17.07 \pm 0.01$ & 300 & OSN $1.5 \mathrm{~m}$ \\
\hline July 30.8682 & $17.46 \pm 0.01$ & 300 & OSN $1.5 \mathrm{~m}$ \\
\hline July 30.8827 & $17.42 \pm 0.01$ & 300 & OSN $1.5 \mathrm{~m}$ \\
\hline July 30.8982 & $17.78 \pm 0.01$ & 300 & OSN $1.5 \mathrm{~m}$ \\
\hline July 30.9053 & $17.93 \pm 0.01$ & 300 & OSN $1.5 \mathrm{~m}$ \\
\hline July 30.9126 & $17.88 \pm 0.02$ & 300 & OSN $1.5 \mathrm{~m}$ \\
\hline July 30.9197 & $17.91 \pm 0.02$ & 300 & OSN $1.5 \mathrm{~m}$ \\
\hline July 31.6736 & $\begin{array}{l}21.48 \pm 0.07 \\
i^{\prime}-\text { passband }\end{array}$ & $300+900$ & HCT $2.01 \mathrm{~m}$ \\
\hline July 30.8644 & $16.68 \pm 0.13$ & 120 & LT $2.0 \mathrm{~m}$ \\
\hline July 30.8705 & $16.76 \pm 0.06$ & 240 & LT $2.0 \mathrm{~m}$ \\
\hline July 30.8798 & $16.74 \pm 0.03$ & 360 & LT $2.0 \mathrm{~m}$ \\
\hline July 30.8909 & $16.90 \pm 0.04$ & 240 & LT $2.0 \mathrm{~m}$ \\
\hline July 30.8939 & $16.92 \pm 0.04$ & 240 & $\mathrm{LT} 2.0 \mathrm{~m}$ \\
\hline July 30.9066 & $17.18 \pm 0.04$ & 360 & LT $2.0 \mathrm{~m}$ \\
\hline July 30.9105 & $17.14 \pm 0.04$ & 240 & LT $2.0 \mathrm{~m}$ \\
\hline July 30.9469 & $\begin{array}{c}17.08 \pm 0.04 \\
I-\text { passband }\end{array}$ & 240 & LT $2.0 \mathrm{~m}$ \\
\hline July 30.8495 & $15.66 \pm 0.01$ & 300 & OSN $1.5 \mathrm{~m}$ \\
\hline July 30.8646 & $16.35 \pm 0.01$ & 300 & OSN $1.5 \mathrm{~m}$ \\
\hline July 30.8791 & $16.34 \pm 0.01$ & 300 & OSN $1.5 \mathrm{~m}$ \\
\hline July 30.8941 & $16.59 \pm 0.01$ & 300 & OSN $1.5 \mathrm{~m}$ \\
\hline July 30.9088 & $16.87 \pm 0.01$ & 300 & OSN $1.5 \mathrm{~m}$ \\
\hline July 30.9232 & $16.80 \pm 0.02$ & 300 & OSN $1.5 \mathrm{~m}$ \\
\hline July 30.9503 & $16.74 \pm 0.01$ & 400 & IAC 80 \\
\hline July 30.9517 & $16.87 \pm 0.03$ & 225 & CTIO $1.3 \mathrm{~m}$ \\
\hline July 30.9623 & $16.97 \pm 0.02$ & 225 & CTIO $1.3 \mathrm{~m}$ \\
\hline July 30.9671 & $17.10 \pm 0.02$ & 225 & CTIO $1.3 \mathrm{~m}$ \\
\hline July 30.9769 & $17.22 \pm 0.02$ & 225 & CTIO $1.3 \mathrm{~m}$ \\
\hline July 31.9167 & $20.75 \pm 0.08$ & $900+1800+2200$ & IAC80 \\
\hline Aug. 01.1963 & $20.73 \pm 0.01$ & $360 \times 6$ & CTIO $1.3 \mathrm{~m}$ \\
\hline Aug. 03.0341 & $\begin{array}{l}22.64 \pm 0.46 \\
J-\text { passband }\end{array}$ & $300 \times 11$ & OSN $1.5 \mathrm{~m}$ \\
\hline July 30.9537 & $16.17 \pm 0.07$ & $45 \times 4$ & CTIO $1.3 \mathrm{~m}$ \\
\hline July 30.9605 & $16.14 \pm 0.08$ & $45 \times 4$ & CTIO $1.3 \mathrm{~m}$ \\
\hline July 30.9639 & $16.55 \pm 0.08$ & $45 \times 4$ & CTIO $1.3 \mathrm{~m}$ \\
\hline July 30.9711 & $16.46 \pm 0.07$ & $45 \times 4$ & CTIO $1.3 \mathrm{~m}$ \\
\hline July 31.0285 & $\begin{array}{l}>19.5 \pm 0.1 \\
K-\text { passband }\end{array}$ & $60 \times 30$ & CTIO $1.3 \mathrm{~m}$ \\
\hline July 30.9538 & $14.73 \pm 0.10$ & $45 \times 8$ & CTIO $1.3 \mathrm{~m}$ \\
\hline July 30.9708 & $15.01 \pm 0.10$ & $45 \times 8$ & CTIO $1.3 \mathrm{~m}$ \\
\hline
\end{tabular}


Table 3. The identification number (ID), $(\alpha, \delta)$ for epoch 2000, standard $V,(B-V),(V-R)$ and $(V-I)$ photometric magnitudes of the stars in the GRB 050730 region.

\begin{tabular}{ccccccc}
\hline \hline ID & $\begin{array}{c}\text { RA(J2000) } \\
(\mathrm{h}: \mathrm{m}: \mathrm{s})\end{array}$ & $\begin{array}{c}\text { Dec(J2000) } \\
(\circ:,:, \prime)\end{array}$ & $\begin{array}{c}V \\
(\mathrm{mag})\end{array}$ & $\begin{array}{c}B-V \\
(\mathrm{mag})\end{array}$ & $\begin{array}{c}V-R \\
(\mathrm{mag})\end{array}$ & $\begin{array}{c}V-I \\
(\mathrm{mag})\end{array}$ \\
\hline 01 & 140810.9 & -034640.2 & 15.15 & 0.41 & 0.23 & 0.73 \\
02 & 140814.6 & -034627.7 & 18.06 & 0.53 & 0.29 & 0.89 \\
03 & 140817.2 & -034636.1 & 18.94 & 1.10 & 0.68 & 1.72 \\
04 & 140819.4 & -034543.9 & 19.02 & 0.56 & 0.35 & 1.07 \\
05 & 140822.1 & -034816.9 & 16.11 & 1.13 & 0.67 & 1.61 \\
06 & 140818.7 & -034914.9 & 15.70 & 0.50 & 0.29 & 0.87 \\
07 & 140818.4 & -034446.8 & 17.72 & 0.76 & 0.45 & 1.18 \\
08 & 140814.5 & -034442.6 & 17.51 & 0.73 & 0.46 & 1.18 \\
09 & 140811.6 & -034436.8 & 17.30 & 0.64 & 0.36 & 1.01 \\
10 & 140826.7 & -034651.9 & 16.56 & 0.71 & 0.41 & 1.07 \\
\hline
\end{tabular}

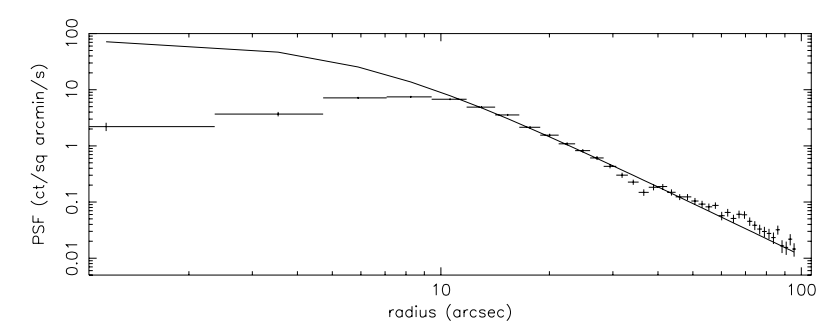

Fig. 1. GRB $050730 \mathrm{X}$-ray afterglow Photon Counting data for $T_{0}+$ $4 \mathrm{ks}$ to $T_{0}+6.6 \mathrm{ks}$. The point spread function and the data diverge at $\sim 10$ arcsec. Only events outside the 10 arcsec region can be used for the analysis.

Source and background regions were extracted using a rectangular aperture for the WT data. The PC data from $T_{0}+4 \mathrm{ks}$ to $T_{0}+24$ ks was "piled-up" due to the intensity of the source. Pileup occurs when more than one photon is collected in CCD frame and they are counted as a single event of higher energy. The main result is an apparent loss of flux from the center of the Point Spread Function (PSF) as shown in Fig. 1 (for a detailed discussion of pile up in XRT see Vaughan et al. 2006). Figure 1 shows the PSF of the XRT and the PC data from $T_{0}+4$ to $T_{0}+6.6 \mathrm{ks}$. Clearly the count rate is diminished inside 10 arcsec and this region of the CCD should not be used for spectra and in addition care must be taken to estimate the true count rate. Annular source regions were used to extract spectra for the piled-up PC data. The radius of the affected inner annulus was determined by fitting the PSF to the data and selecting regions where the data are well fit by the PSF (as shown in Fig. 1). Circular source regions were used to extract the spectra after $27 \mathrm{ks}$ since the rate had fallen below the critical level of 0.6 counts $\mathrm{s}^{-1}$. In addition, the data were affected by bad CCD columns and the rate was corrected (A. Beardmore \& K. Page, private communication).

\subsubsection{XMM-Newton observations}

Target of Opportunity observations with XMM-Newton ${ }^{7}$ of the region around the BAT error box of GRB 050730 were triggered in revolution 1033 (Observation ID. 0164571201) (Schartel 2005). The observations started at 03:00:08 UT on 2005 July 31, i.e., $25 \mathrm{ks}$ after the burst. The EPIC/MOS1, EPIC/MOS2 and EPIC/pn CCD cameras were operated in the Prime Full Window Mode for a total exposure time of $33.2 \mathrm{ks}$ for the EPIC/MOS and

\footnotetext{
7 Based on observations obtained with XMM-Newton, an ESA science mission with instruments and contributions directly funded by ESA Member States and NASA.
}

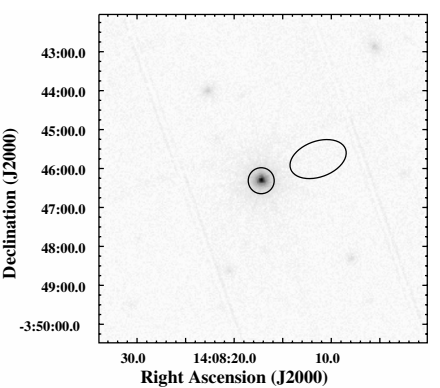

Fig. 2. XMM-Newton raw EPIC image in the $0.45-8.0 \mathrm{keV}$ energy band of the region around the Swift-BAT error box of GRB 050730. The X-ray afterglow of GRB 050730 is the bright central source in this image. The circle and ellipse indicate the spatial regions used for source and background extraction, respectively.

$25.7 \mathrm{ks}$ for the EPIC/pn. The EPIC/MOS1 observations used the Medium optical blocking filter, while the EPIC/MOS2 and the EPIC/pn observations used the Thin1 filter.

The XMM-Newton pipeline products were processed using the XMM-Newton Science Analysis Software (SAS version 6.1.0) and the calibration files from the Calibration Access Layer that were available on 2005 November 24. Time intervals with high background were discarded (i.e., count rates in the background-dominated $10-12 \mathrm{keV}$ energy range $\geq 0.4 \mathrm{cnts} \mathrm{s}^{-1}$ for the EPIC/MOS or $\geq 1.2 \mathrm{cnts} \mathrm{s}^{-1}$ for the EPIC/pn). The resulting exposure times were $26.4 \mathrm{ks}, 25.5 \mathrm{ks}$, and $17.9 \mathrm{ks}$ for the EPIC/MOS1, EPIC/MOS2 and EPIC/pn observations respectively.

In order to search for the X-ray afterglow of GRB 050730, we extracted raw XMM-Newton EPIC/MOS1, EPIC/MOS2 and EPIC/pn images in the $0.45-8.0 \mathrm{keV}$ band with a pixel size of $2^{\prime \prime}$. These images show a bright X-ray point-source within the Swift-BAT error box of GRB 050730, as shown in the merged EPIC image in Fig. 2. The location of this X-ray source, at $\operatorname{RA}(\mathrm{J} 2000)=14^{\mathrm{h}} 8^{\mathrm{m}} 17.2^{\mathrm{s}}, \operatorname{Dec}(\mathrm{J} 2000)=-3^{\circ} 46^{\prime} 18^{\prime \prime} \cdot 6$, is coincident with the optical transient of GRB 050730 (Sota et al. 2005), thus identifying of the X-ray afterglow of GRB 050730. The XMM-Newton EPIC/MOS1, EPIC/MOS2, and EPIC/pn observations of this source detect a total of $7350 \pm 100 \mathrm{cnts}, 7700 \pm$ $100 \mathrm{cnts}$ and $19000 \pm 140 \mathrm{cnts}$ respectively. We would like to emphasize that this X-ray source is well away from the gaps in the EPIC/MOS and EPIC/pn CCDs, thus allowing a reliable determination of the count rate.

\section{Results}

\subsection{NIR-optical photometric light-curves}

The optical and NIR light-curves of the afterglow of GRB 050730 in the $B, V, r^{\prime}, R, i^{\prime}, I$ and $J$ passbands are shown in Fig. 3. The observations presented in this figure are supplemented by photometric measurements of the afterglow published in GCN circulars (Fig. 3 and the caption). The supplementary data were also calibrated using the secondary standards tabulated in Table 3.

The light-curves in Fig. 3 are presented relative to the GRB trigger time $\left(T_{0}=2005\right.$ July $\left.30.8322 \mathrm{UT}\right)$. Although sparsely sampled, it appears that the $B$ and $V$ light-curves show similar behaviour up to 0.1 days post-burst. Near achromatic variability is clearly present in the afterglow light-curves of all the pass bands. However, the light-curves do not display the correlated flaring behavior observed in the early X-ray afterglow 


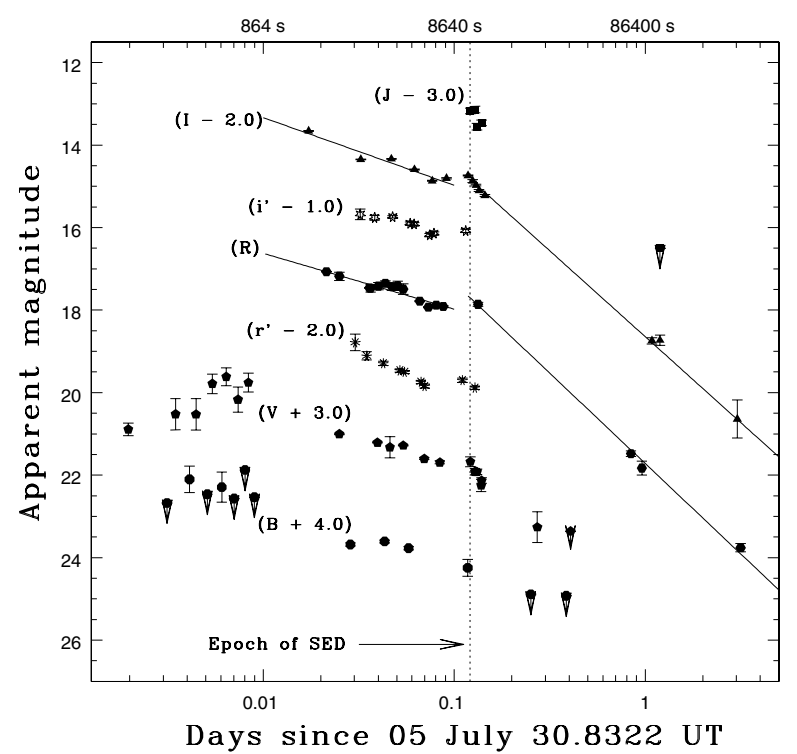

Fig. 3. Optical light-curves of GRB 050730 afterglow in $B, V, r^{\prime}, R, i^{\prime}, I$ and $J$ pass bands. Marked vertical offsets are applied to avoid overlapping of data points in different passbands. The solid curves are the linear least square best fitted power-law relations for $R$ and $I$ passbands. Vertical dotted line shows the epoch of NIR-optical SED shown in Fig. 4. Data presented in this work (Table 2) and supplementary data (Bhatt \& Sahu 2005; Blustin et al. 2005; Burenin et al. 2005; Damerdji et al. 2005; Gomboc et al. 2005; Haislip et al. 2005; and Kannappan et al. 2005) are combined in this figure.

light-curve (Figs. 5 and 10). The values of flux decay indices using a single power-law fit $\left(f_{v} \propto t^{\alpha_{1}}\right)$ to the $R$ and $I$ data points between 0.01 to 0.1 day after the burst are $\alpha_{1}=-0.54 \pm 0.05$ and $-0.66 \pm 0.11$ respectively. Similar early time decay slopes were also obtained from the $B, V, r^{\prime}$ and $i^{\prime}$ afterglow light curves over a similar time scale but the $R$ and $I$ band light curves gave the best fits. The $J, I$ and $V$ light-curves, although sparsely sampled, show a bump followed by a considerable steepening at about 0.1 day $(8.6 \mathrm{ks})$. The $R$ and $I$ light-curves after 0.1 day were fit by power-law index values $\left(f_{v} \propto t^{\alpha_{2}}\right)$ of $\alpha_{2}=-1.75 \pm 0.05$ and $-1.66 \pm 0.07$ respectively. Late time $V$ and $J$ afterglow light curves also have $\alpha_{2} \sim-2$. The variability in the multi-band afterglow light-curves of GRB 050730 does not allow a reasonable fit for the generic broken power-law model (Beuermann et al. 1999). Therefore, we conclude that the early time light-curve decay slope is $\alpha_{1}=-0.60 \pm 0.07$ and after a break time around 0.1 day, the weighted mean value of $\alpha_{2}$ is $-1.71 \pm 0.06$ using $R$ and $I$ band data.

\subsection{NIR-optical Spectral Energy Distribution}

The Spectral Energy Distribution (SED) of the afterglow of GRB 050730 was generated at $2.9 \mathrm{~h}(10.4 \mathrm{ks})$ after the burst trigger. This epoch was chosen to avail of simultaneous ANDICAM data in the $V, I, J$ and $K$ pass-bands. The SED from NIR to optical frequencies is presented in Fig. 4. The reddening map provided by Schlegel et al. (1998) indicates a small value of $E(B-V)=0.05 \mathrm{mag}$ for the Galactic interstellar extinction in the direction of the burst. We used the standard Galactic extinction reddening curve given by Mathis (1990) to convert apparent magnitudes into fluxes, with the effective wavelengths and normalizations from Bessel et al. (1998) for $V, R, I, J, K$ passbands. Due to the high redshift $(z \sim 3.97)$ of the burst, the Ly- $\alpha$ break lies between the $R$ and $I$ pass-bands, making NIR

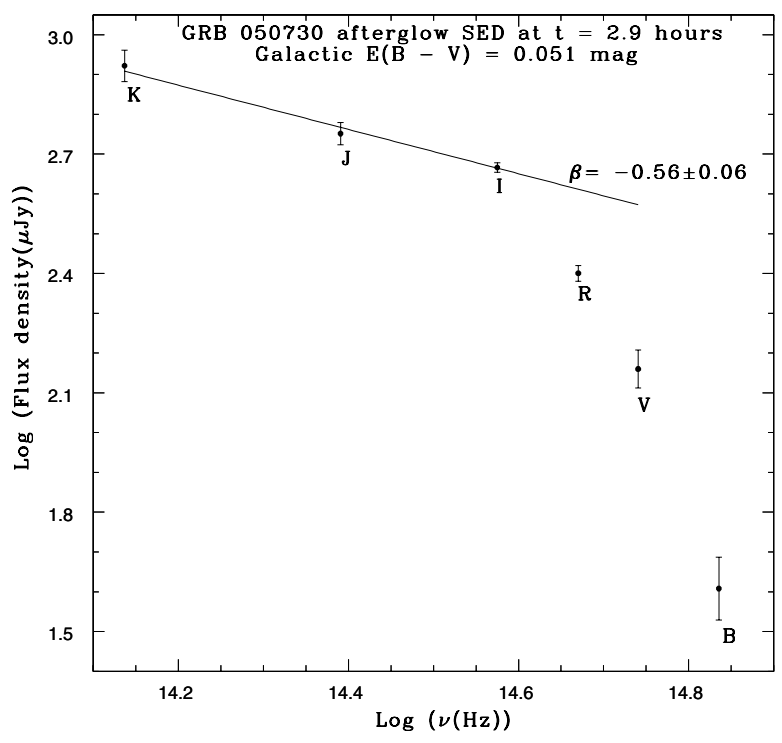

Fig. 4. Spectral energy distribution (SED) of GRB 050730 at $t=2.9 \mathrm{~h}$ (10.4 ks) after the burst in the NIR and optical. The magnitudes are corrected for the galactic extinction of $E(B-V)=0.05$ mag in the burst direction. The epoch was chosen at the simultaneous $V, I, J$ and $K$ observations using ANDICAM on the $1.3 \mathrm{~m}$ telescope at CTIO. The effect of damped Ly- $\alpha$ due to the high redshift of the burst $(z \sim 3.97)$ is seen in form of considerable drop in the observed flux values between $I$ and $R$ data points.

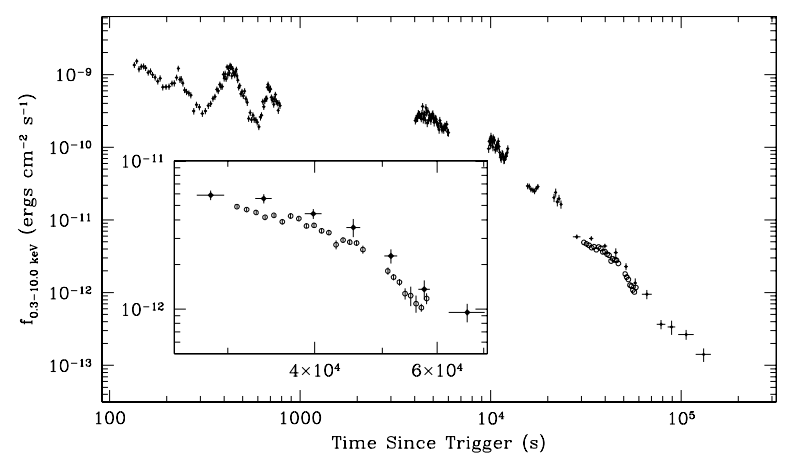

Fig. 5. The Swift/XRT (closed circles) and XMM-Newton (open circles) light-curve of GRB 050730. Superimposed variability is clearly visible over most of the light-curve. The inset shows the correlation between Swift/XRT and XMM-Newton results.

frequencies essential to determine the correct spectral index of the burst. Since the $R$ passband flux is also partially suppressed by the Ly- $\alpha$ break, we used only $I, J, K$ data to determine the spectral index at the epoch of the SED. If no spectral break occurs, the SED is generally described as a power law: $F(v) \propto v^{\beta}$, where $\beta$ is the spectral index. The derived spectral index is $\beta=-0.56 \pm 0.06$ (Fig. 4). Spectroscopic observations of the afterglow taken $\sim 3 \mathrm{~h}$ after the burst by Starling et al. (2005) show negligible extinction in the host.

\subsection{Swift data analysis}

\subsubsection{Light-curve analysis}

The XRT light-curve is complex with flares in the early WT data at $\sim 250 \mathrm{~s}, \sim 420 \mathrm{~s}$ and $\sim 650 \mathrm{~s}$ (Fig. 5, see also Starling et al. 2005). These early time flaring behaviors with rising or decaying slope index values $\sim-6$ are unusual and are not expected by 
Table 4. Spectral fit parameters from Swift and XMM-Newton data analysis. The columns list the time interval over which the spectra were fit, the column density at the redshift of the host $z=3.967$, the photon index, observed flux, unabsorbed flux, $\chi^{2}$ and degrees of freedom. The column density for the $S$ wift/XRT data was obtained by fitting the time intervals simultaneously while allowing the photon index to vary. Errors are quoted at the $90 \%$ level for each parameter of interest.

\begin{tabular}{|c|c|c|c|c|c|c|}
\hline $\begin{array}{c}\text { Time interval } \\
\text { (seconds since Trigger) }\end{array}$ & $\begin{array}{l}\text { Column density } N_{\mathrm{H}} \\
\times 10^{21} \mathrm{~cm}^{2}\end{array}$ & $\begin{array}{l}\text { Power-law index } \\
\Gamma\end{array}$ & $\begin{array}{c}f_{\mathrm{obs}} \\
\operatorname{ergs~} \mathrm{cm}^{-2} \mathrm{~s}^{-1}\end{array}$ & 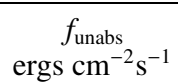 & $x^{2}$ & d.o.f \\
\hline$\ddagger 132-232$ & \multirow{3}{*}{$11.4_{-7.0}^{+7.0}$} & $1.30 \pm 0.07$ & $9.7 \times 10^{-10}$ & $10 \times 10^{-10}$ & 120 & 92 \\
\hline $232-332$ & & $1.7 \pm 0.13$ & $4.4 \times 10^{-10}$ & $5.1 \times 10^{-10}$ & 80.6 & 58 \\
\hline $332-432$ & & $1.50 \pm 0.09$ & $7.3 \times 10^{-10}$ & $7.9 \times 10^{-10}$ & 107.6 & 79 \\
\hline $432-532$ & \multirow{3}{*}{$3.0^{\dagger}$} & $1.56_{-0.13}^{+0.10}$ & $7.3 \times 10^{-10}$ & $7.7 \times 10^{-10}$ & 97.9 & 84 \\
\hline $532-632$ & & $1.87 \pm 0.06$ & $4.4 \times 10^{-10}$ & $4.8 \times 10^{-10}$ & 96.5 & 94 \\
\hline $632-790$ & & $1.84 \pm 0.05$ & $4.5 \times 10^{-10}$ & $4.9 \times 10^{-10}$ & 86.7 & 95 \\
\hline $4-6.6 \mathrm{ks}$ & \multirow[b]{2}{*}{$12.2_{-3.0}^{+3.5}$} & $1.60 \pm 0.05$ & $2.2 \times 10^{-10}$ & $2.4 \times 10^{-10}$ & 148.6 & 148 \\
\hline $9.7-12.4 \mathrm{ks}$ & & $1.73 \pm 0.07$ & $1.0 \times 10^{-10}$ & $1.1 \times 10^{-10}$ & 89.9 & 111 \\
\hline $15.5-23.9 \mathrm{ks}$ & \multirow{3}{*}{$11.5 \pm 6.0$} & $1.73_{-0.09}^{+0.10}$ & $2.4 \times 10^{-11}$ & $2.8 \times 10^{-11}$ & 34.6 & 39 \\
\hline $27-47 \mathrm{ks}$ & & $1.84_{-0.13}^{+0.09}$ & $4.5 \times 10^{-12}$ & $4.8 \times 10^{-12}$ & 34.1 & 41 \\
\hline $50-140 \mathrm{ks}$ & & $1.75 \pm 0.14$ & $4.8 \times 10^{-13}$ & $5.7 \times 10^{-13}$ & 28 & 20 \\
\hline${ }^{\S} 30-56 \mathrm{ks}$ & $6.7_{-1.9}^{+1.9}$ & $1.91 \pm 0.03$ & $3.0 \times 10^{-12}$ & $3.4 \times 10^{-12}$ & 388.9 & 401 \\
\hline $30-40 \mathrm{ks}$ & $7.1_{-24}^{+2.9}$ & $1.87 \pm 0.04$ & $4.1 \times 10^{-12}$ & $4.6 \times 10^{-12}$ & 256.9 & 302 \\
\hline $40-56 \mathrm{ks}$ & $5.5_{-3.0}^{+3.4}$ & $1.96 \pm 0.04$ & $2.0 \times 10^{-12}$ & $2.3 \times 10^{-12}$ & 200.2 & 202 \\
\hline
\end{tabular}

${ }^{\ddagger}$ Swift epochs; ${ }^{\S}$ XMM-Newton epochs $; \dagger 99 \%$ confidence Upper limit.

simple synchrotron fireball model (Sari et al. 1998). A flare is also detected in PC data at $\sim 4500 \mathrm{~s}$. The early data cannot be fit by a single power-law due to the flares. The light-curve after $15 \mathrm{ks}$ can be fit by a power-law but shows evidence of variability in some time intervals with an overall decay index of $-2.5 \pm 0.15$, in agreement with Perri et al. (2005). The XRT light curve is presented together with the $R$ and $V$ band light curves in Fig. 10 for comparison purposes.

\subsubsection{Spectral analysis}

Spectra were extracted from the XRT data for 11 time intervals and the results are presented in Table 4 . The spectra were fit with an absorbed power-law model including galactic column density of $N_{\mathrm{H}}^{\mathrm{GAL}}, 3.2 \times 10^{20} \mathrm{~cm}^{-2}$ (Dickey \& Lockman 1990) and intrinsic absorption in the host at $z=3.967$. Several spectra were fit simultaneously to obtain an individual column density and to allow the power-law indices to vary independently (Table 4). A simultaneous spectral fit of the first three WT spectra from $T_{0}+132 \mathrm{~s}$ to $T_{0}+432 \mathrm{~s}$ and a contour plot of the column density at $z=3.967$ versus the photon index $(\Gamma)$ of the first interval are shown in Fig. 6. Combining these three intervals yields a column density of $(11.4 \pm 7) \times 10^{21} \mathrm{~cm}^{-2}$. Only a $99 \%$ confidence upper limit of $3.0 \times 10^{21} \mathrm{~cm}^{-2}$ could be obtained for the column density using the subsequent three WT spectra in the intervals from $T_{0}+$ $432 \mathrm{~s}$ to $T_{0}+790 \mathrm{~s}$ (Table 4 ).

The simultaneous fit of the first two PC spectra from $T_{0}+4 \mathrm{ks}$ to $T_{0}+12.4 \mathrm{ks}$ and a contour plot of the column density at $z=$ 3.967 versus the photon index of the first interval are shown in Fig. 7. A tabulation of all the spectral fits to the XRT data and intervals is listed in Table 4.

The photon index evolves in afterglow from an early value of $\sim 1.30$ to $\sim 1.75$ (or $\sim 1.9$ using XMM-Newton data (see Sect. 3.4.2)). Starling et al. (2005) reported an excess column density in the first half of the WT observation along with an abrupt change at $T_{0}+500 \mathrm{~s}$ and an increase in the photon index consistent with these results.

It should be noted that the $\chi^{2} /$ d.o.f. values in Table 4 for the WT data from $T_{0}+132$ to $T_{0}+423 \mathrm{~s}$ indicate that the absorbed
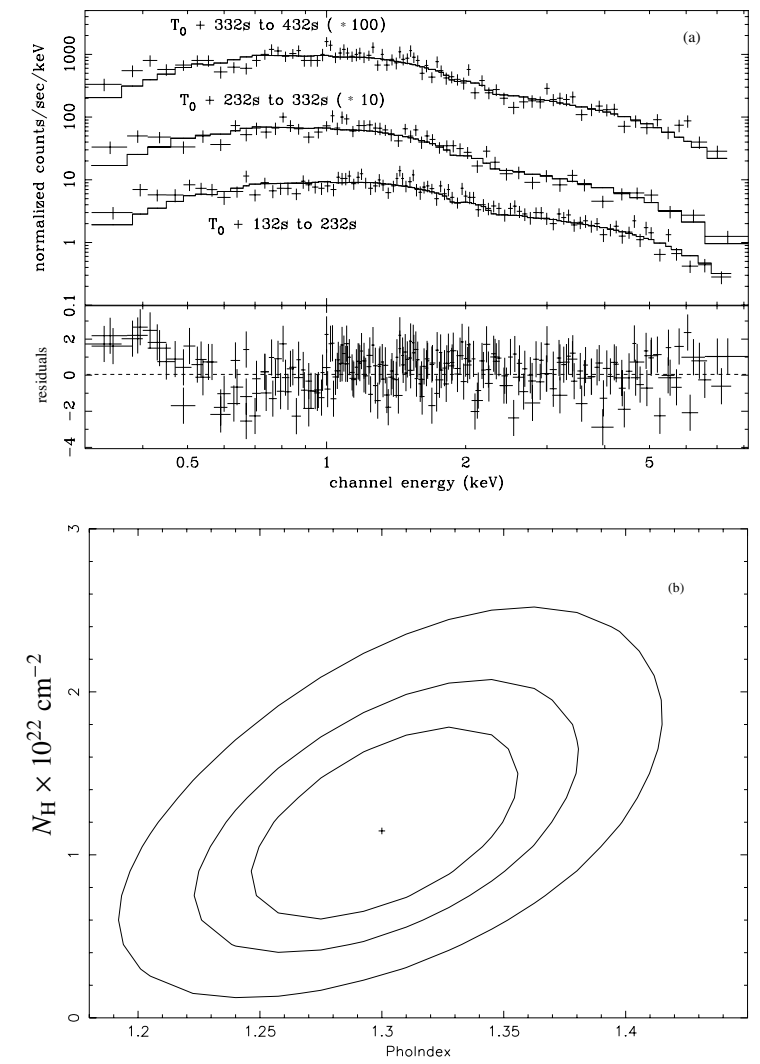

Fig. 6. a) Simultaneous spectral fit for WT intervals from $T_{0}+132 \mathrm{~s}$ to $T_{0}+432 \mathrm{~s}$, best fit parameters are listed in Table 4 (spectra are offset for presentation purposes) and b) Two dimensional confidence contours at $68.3 \%, 90 \%$ and $99 \%$ for $N_{\mathrm{H}}$ of the absorber component at the burst redshift and the photon index for the first interval.

power-law may not be the best model (Fig. 6). A $\chi^{2} /$ d.o.f. value of $310 / 228$ is achieved by simultaneously fitting an absorbed power-law model to these data resulting in the large uncertainities in the intrinsic $N_{\mathrm{H}}$ values. The data in the interval $T_{0}+132$ to $T_{0}+423 \mathrm{~s}$ were also fit with a broken power-law model and 

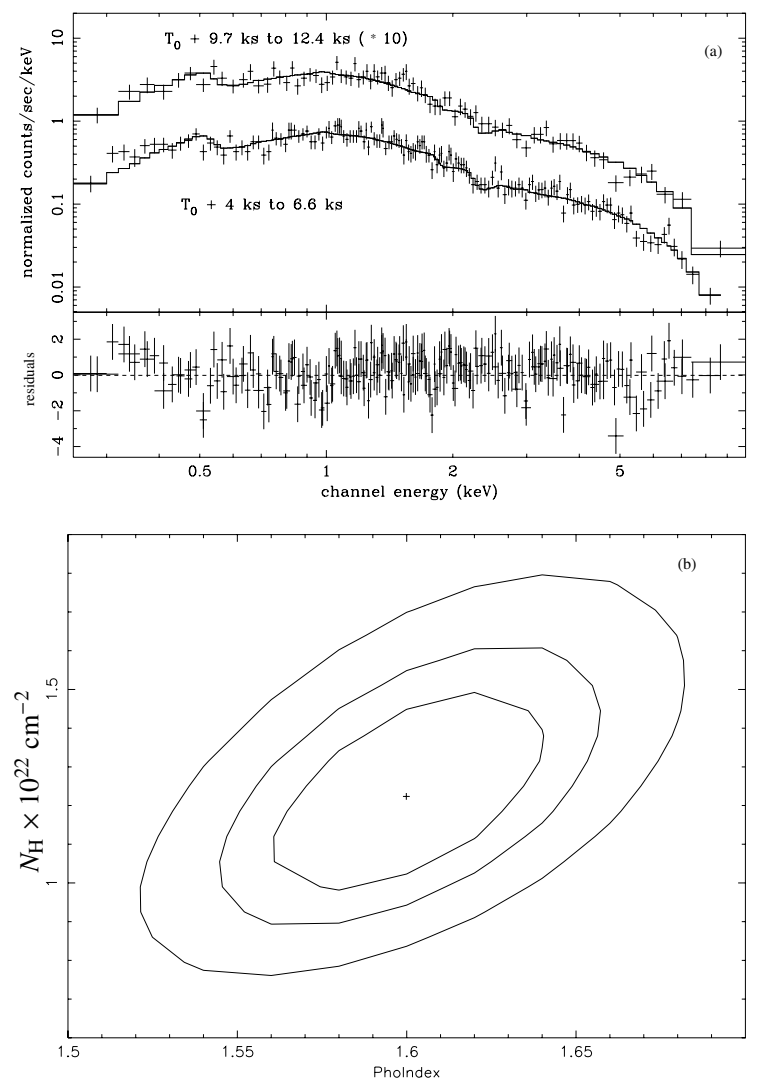

Fig. 7. a) Simultaneous spectral fit for PC intervals in two intervals, $T_{0}+4 \mathrm{ks}$ to $T_{0}+12.4 \mathrm{ks}$, best fit parameters are listed in Table 4 (spectra are offset for presentation purposes) and b) Two dimensional confidence contours at $68.3 \%, 90 \%$ and $99 \%$ for $N_{\mathrm{H}}$ of the absorber component at the burst redshift and the photon index for the first interval.

an absorbed power-law with a black body component and the $\chi^{2} /$ d.o.f. values were $264.2 / 227$ and $265.2 / 226$ respectively.

At later epochs after $T_{0}+432 \mathrm{~s}$ the $\chi^{2} /$ d.o.f. values for the simultaneous fits are closer to unity. The absorbed power-law fit from $T_{0}+432$ to $T_{0}+790 \mathrm{~s}$ yields $\chi^{2} /$ d.o.f. of $198.9 / 184$. The $\chi^{2} /$ d.o.f. for the epoch $T_{0}+4 \mathrm{ks}$ to $T_{0}+6.6 \mathrm{ks}$ is $232 / 230$ and the value for the epoch $T_{0}+15.5 \mathrm{ks}$ to $T_{0}+140 \mathrm{ks}$ the value is $99 / 102$. The results for the absorbed power-law fits are presented in Table 4 for all epochs to allow a comparison between the early and late time $S$ wift/XRT spectra and the XMM-Newton data. The evolution of the power law index and the column density are presented with the X-ray, $R$ and $V$ light curves in Fig. 10.

\subsection{XMM-Newton data analysis}

\subsubsection{Light-curve analysis}

We have extracted background-subtracted light-curves in the $0.3-10.0 \mathrm{keV}$ energy band of the X-ray afterglow of GRB 050730 using the circular source region of radius $20^{\prime \prime}$ shown in Fig. 2. For the background region, an elliptical region 3 times the area of the source region and located at the same $\mathrm{Y}$ coordinate of the EPIC/pn CCD as the source region was used (also shown in Fig. 2). The EPIC/pn and combined EPIC/MOS background-subtracted light-curves with time bins of $1 \mathrm{ks}$ are shown in Fig. 5. The XMM-Newton light-curve shows an agrees with $S$ wift light-curve in the nature of decay as shown in the inset of Fig. 5.
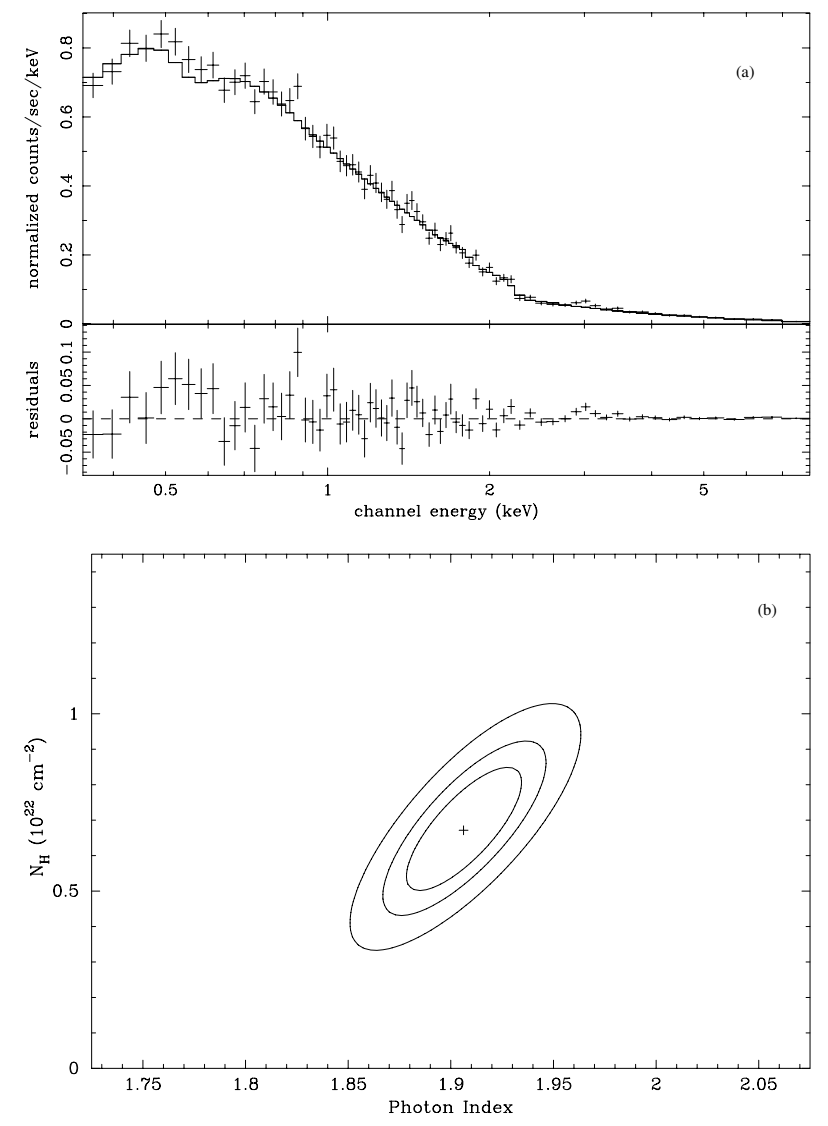

Fig. 8. a) The EPIC/pn spectrum for the total exposure time where the best fit parameters are listed in Table 4. b) Two-dimensional confidence contours at $68.3 \%, 90 \%$, and $99 \%$ for $N_{\mathrm{H}}$ of the absorber component at the burst redshift and $\Gamma$ of the spectral fit to the total exposure time EPIC/pn Illegal variable name. spectrum of GRB 050730.

\subsubsection{Spectral analysis}

Source and background EPIC/MOS and EPIC/pn spectra of the X-ray afterglow of GRB 050730 were obtained from the same spatial regions as those used for the light-curve extraction (Fig. 2). When the background spectrum is scaled to the area of the source spectrum, it contributes only $\sim 1 \%$ to the net source count rate, i.e., the background contribution to the observed spectrum is almost negligible. The backgroundsubtracted EPIC/pn spectrum is shown in Fig. 8. The EPIC/MOS spectra (not shown here) show similar spectral behavior, but at lower signal-to-noise ratios than the EPIC/pn spectrum, so we concentrate on the spectral analysis of this later one.

The spectra were fit by an absorbed power-law model with two absorption components: an absorber component at the burst redshift of $z=3.967$ and a Galactic absorber component whose hydrogen column density, $N_{\mathrm{H}}^{\mathrm{Gal}}$, has been fixed at $3.2 \times 10^{20} \mathrm{~cm}^{-2}$ (Dickey \& Lockman 1990). The same model was fit to the XRT spectra using XSPEC (Arnaud 1996). The spectral fit was carried out by folding the absorbed power-law model spectrum through the EPIC/pn response matrix, and comparing the modeled spectrum to the observed EPIC/pn spectrum in the $0.35-8.0 \mathrm{keV}$ energy range using the $\chi^{2}$ statistic. To ensure that the $\chi^{2}$ statistic correctly can be used, the spectrum was binned to have a $S / N>5$ per spectral bin.

The best-fit model, shown in Fig. 8, provides an excellent fit to the EPIC/pn spectrum, with a reduced $\chi^{2}$ close to unity, $\chi^{2} /$ d.o.f. $=0.98$. The quality of the spectral fit is further 

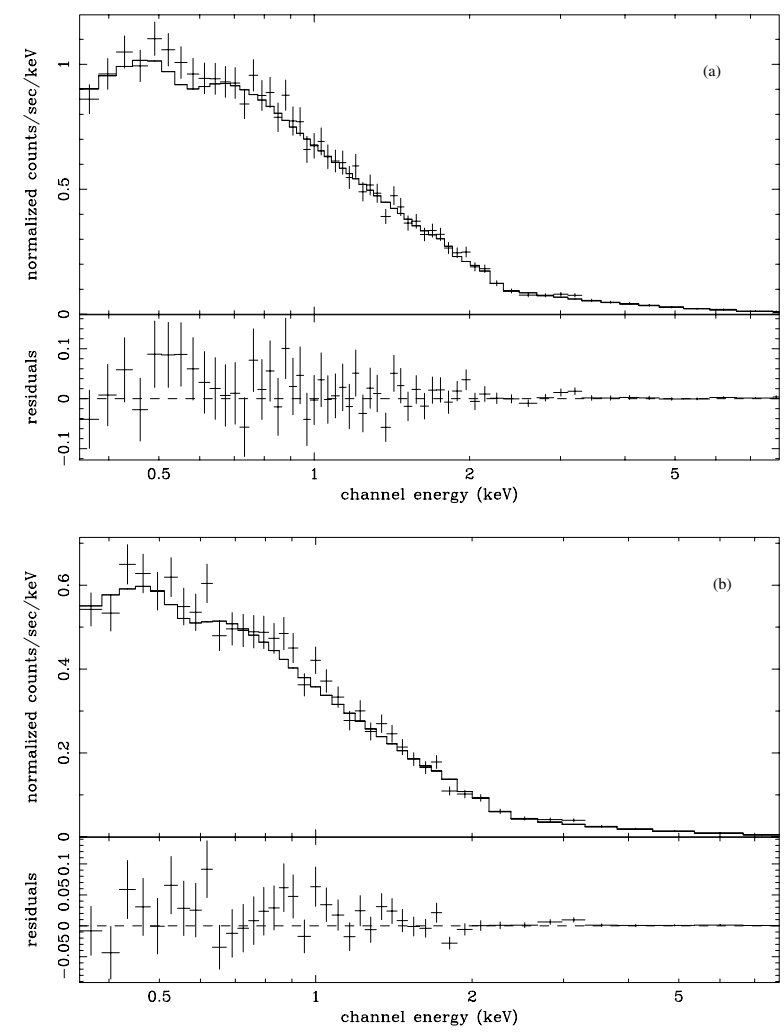

Fig. 9. a) The EPIC/pn spectrum for the first a) and second b) exposure times listed in Table 4.

illustrated by the plot of confidence contours as a function of $\Gamma$ and $N_{\mathrm{H}}$ shown in Fig. 8. The fitted model parameters with $90 \%$ confidence level are listed in the last row of Table 4; the spectral photon index, $\Gamma$, is $1.91 \pm 0.03$, and the hydrogen column density of the absorber component at the burst redshift is $(6.7 \pm 1.9) \times 10^{21} \mathrm{~cm}^{2}$. The residuals shown in A look at the fit residuals in Fig. 8 suggest the presence of weak features in the spectrum. We therefore add to the absorbed power-law a thermal model component (the MEKAL model in XSPEC) and Gaussian lines at different energies, but in all cases the corresponding $\chi^{2} /$ d.o.f. departed from unity. We confidently conclude that the X-ray emission of the afterglow of GRB 050730 can be described by an absorbed power-law model with no significant contribution of thermal emission. The EPIC/pn spectrum of GRB 050730 does not show any noticeable emission line and its spectral shape is suggestive of power-law models, representing synchrotron emission from a population of relativistic electrons.

We have also searched for spectral variability throughout the XMM-Newton EPIC/pn observation of GRB 050730. The total useful exposure time (after subtraction of periods of highbackground) was divided into 2 segments including the initial $8.2 \mathrm{ks}$ and the final $9.6 \mathrm{ks}$ of the total exposure time. The corresponding EPIC/pn spectra and best-fit models are shown in Fig. 9. The quality of these fits is not better than the combined spectral fit based on the value of $\chi^{2}$ tabulated in Table 4. The other parameters of these best-fit models are also listed in Table 4. An inspection of these parameters suggests a marginal steepening of the spectral index.

\section{Discussion}

We have presented early time optical and NIR photometry of the afterglow of GRB 050730 and millimeter observations. The

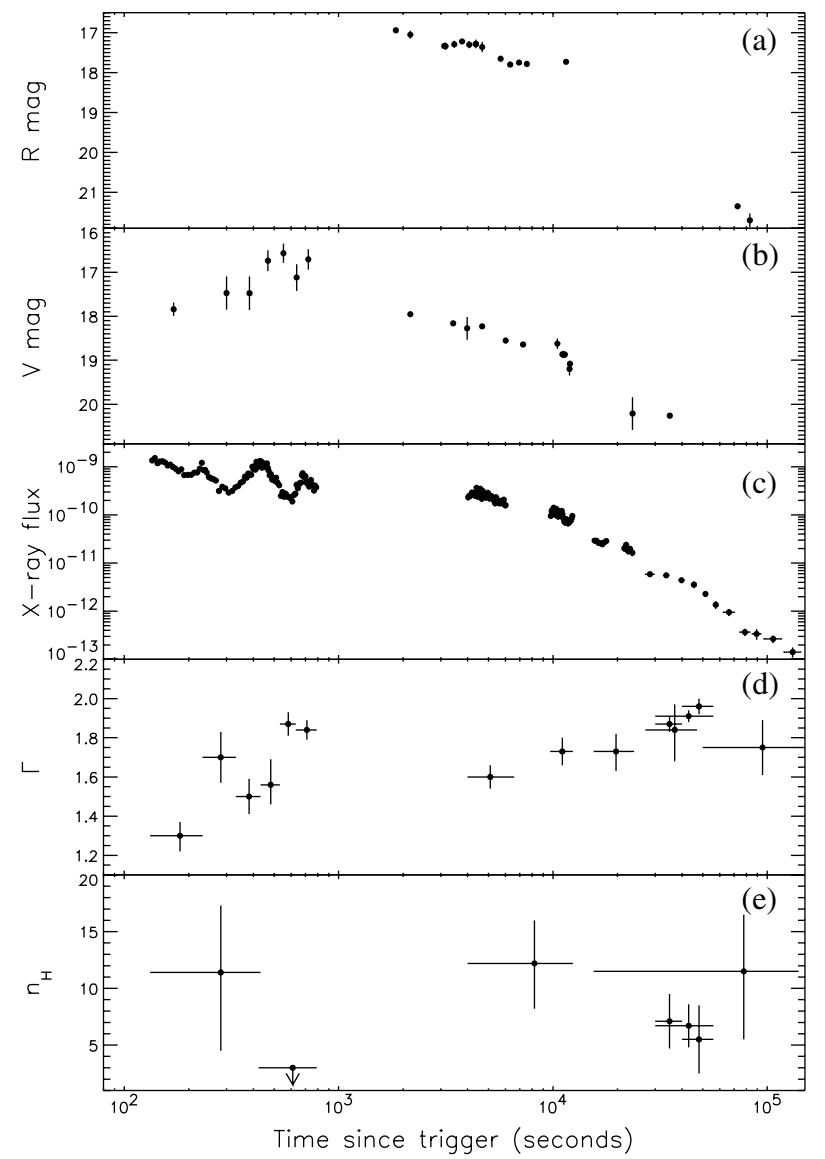

Fig. 10. The $R$ and $V$ band and X-ray light-curves of GRB 050730 are shown in panels a), b) and c) respectively. The temporal evolution of parameters column density $N_{\mathrm{H}}$ (in units of $\times 10^{21} \mathrm{~cm}^{-2}$ ) and photon index derived from the X-ray afterglow spectrum of GRB 050730 are shown in panels $\mathbf{d}$ ) and $\mathbf{e}$ ). The overall nature of the light curves shows variability but the poor sampling of the optical data prevents correlation test.

optical afterglow light-curves (Figs. 3 and 10) show early time variability similar to that observed in GRB 000301C (Sagar et al. 2000) and GRB 021004 (Pandey et al. 2003). X-ray afterglow observations of GRB 050730 from Swift and XMM-Newton (Fig. 5) also show variability (Figs. 5 and 10). The observed $\mathrm{X}$-ray and optical afterglow variability seems to be uncorrelated in the very early phases $(t<0.01$ day) of the light-curves. The nature of the early X-ray flaring and its correlation with BAT light-curves has been discussed (Burrows et al. 2006; Nousek et al. 2006) and may indicate ongoing central engine activity superimposed on a slowly decaying initial afterglow phase. The observed flaring behavior can be explained in terms of one of the theoretical models (King et al. 2005; Perna et al. 2005; Proga \& Zhang 2006).

X-ray afterglow results from XMM-Newton, taken $\sim 25 \mathrm{ks}$ after the burst, show a decay similar to that of the Swift/XRT light-curve as shown by the inset of Fig. 5. The Swift/XRT temporal and spectral analysis are in agreement with those of the XMM-Newton (Table 4). The X-ray and optical light-curves can are given in Fig. 10. The X-ray afterglow light-curve $15 \mathrm{ks}$ after the burst shows an overall temporal flux decay index of $-2.5 \pm 0.15$ which is steeper than the late time optical temporal decay index. Temporal evolution of $\Gamma$ is clearly observed from the first to the last epoch and the value of column density is similar except for a sudden drop around $500 \mathrm{~s}$ after the burst, as 
initially reported by Starling et al. (2005). This result might resemble the variable column density seen in the case of prompt emission of GRB 000528 (Frontera et al. 2004).

The averaged temporal flux decay indices of $\alpha_{1}=-0.60 \pm$ 0.07 and $\alpha_{2}=-1.71 \pm 0.06$ are derived based on the considerable steepening of the $R$ and $I$ data points around 0.1 day (8.6 ks) after the burst. The $S$ wift light-curve after 0.1 day can be fit by a power-law decay index $\alpha_{2}=-2.5 \pm 0.15$, steeper than that derived from the optical observations. The spectral in$\operatorname{dex} \beta=-0.56 \pm 0.06$ is determined from $I, J$ and $K$ pass-band observations at $\sim 10.4 \mathrm{ks}$ after the burst. The value of $\beta$ derived from the X-ray photon index value (Table 4 ) at a similar epoch is $-0.73 \pm 0.07$ which is statistically in agreement with the opticalNIR value, and indicates that the cooling break frequency $v_{\mathrm{c}}$ does not lie between optical and X-ray frequencies at that epoch. The derived values of $\alpha_{2}$ and $\beta$ from optical and X-ray frequencies, in the slow-cooling ISM jet model predictions (Sari et al. 1999; Rhoads 1999), rules out the possibility that $v_{\mathrm{c}}$ is below the NIR frequencies. The location of $v_{c}$ above observed X-ray frequencies at the time of the SED gives the electron energy in$\operatorname{dex} p \sim 2.3$, in agreement with the value of $\alpha_{2}$ derived from the X-ray light-curve. Such a high value of $v_{\mathrm{c}}$ indicates relatively low values for the post shock magnetic field energy $\epsilon_{B}$ and the ambient density (Sari et al. 1999). The location of $v_{\mathrm{c}}$ above the $\mathrm{X}$-ray the around 0.1 day $(8.6 \mathrm{ks})$ after the burst, and no considerable evolution of X-ray spectral index (see Table 4) from 0.1 until 1.5 day $(8.6 \mathrm{ks}$ to $130 \mathrm{ks}$ ) after the burst, indicates that the observed break in the optical light-curves is a jet-break. An early jet-break is also reported for the high redshift burst, GRB 050319 (Cusumano et al. 2006) but not in the case of the highest redshift burst, GRB 050904 (Tagliaferri et al. 2005).

The lower value of $\alpha_{2}$ at optical frequencies with respect to the X-ray regime is inconsistent with predictions from the standard fireball model (Sari et al. 1999). The possibility of a contribution from the host galaxy or an associated supernova to the observed lower value of $\alpha_{2}$ at optical frequencies can be ruled out considering the high redshift of the burst. Apart from an observed variability around 0.1 day after the burst, the $R$ and $I$ afterglow late time light-curves do not show further variability. Other plausible explanations for the observed shallower value of $\alpha_{2}$ at optical frequencies are in terms of modified afterglow models including: refreshed shocks or fluctuations in the external media. In refreshed shock models the fluctuations in the observed flux are expected at both frequencies, but lack of X-ray observations at later epochs does not allow us to constrain the observed flatness at optical frequencies in terms of the model. In the case of the model where the fireball encounters regions of enhanced density (Lazzati et al. 2002; Nakar et al. 2003), the afterglow flux is supposed depend on the external density for the frequencies below $v_{\mathrm{c}}$. In the present case, the location of $v_{\mathrm{c}}$ above observed $\mathrm{X}$-ray frequencies at very early epochs $(\sim 0.1$ day) does not allow this interpretation of the observed flatness at optical frequencies. The possibility of the two component jet model (Berger et al. 2003) can not be ruled out for the observed difference between the $\alpha_{2}$ values at the two frequencies although at radio frequencies no significant observations were found (van der Horst \& Rol 2005a,b). The sparse temporal coverage and the lack of observations 3 days after the burst however, do not distinguish between the various explanations for the observed discrepancy between the values of $\alpha_{2}$ values at optical and X-ray frequencies.

The interpretation of the observed steepening in the afterglow around 0.1 days as a jet-break in terms of the ISM jet model show $\alpha_{2} \sim p$, but the observed value of $\alpha_{1}$ is smaller than expected when compared with the closure relation $\alpha_{1}=3 \beta / 2$. The observed early-time superimposed variability in the form of a flatter value of $\alpha_{1}$ might imply a set of energy-injection episodes (Zhang \& Mészáros 2002) followed by late-time activity from the central engine. For a Poynting-flux-dominated continuous energy injection, Zhang \& Mészáros (2002) assume that the source luminosity $L(t) \propto t^{q}$, where $q$ is the temporal index, and influences the observed light-curve through energy injections for $q>-1$. The value of $q$ is related to the observed $\alpha$ and $\beta$ with a closure relation as long as the observed frequencies are in same spectral region. In the case of $v_{\mathrm{c}}$ being above $\mathrm{X}$-ray frequencies, $\alpha, \beta$ and $q$ are related as $\alpha=(1-q / 2) \beta+q+1$ (Zhang \& Mészáros 2002). The fact that the observed values of $\alpha_{1}$ and $\beta$ are in agreement with the above closure relation with $q>-1$, supports the explanation that the observed flatter value of $\alpha_{1}$ being caused by the early-time light-curve being dominated by energy injection episodes. However, in order to understand the observed variability in terms of the energy injection episodes, detailed modeling is required as in the case of GRB 010222 (Björnsson et al. 2002) and GRB 021004 (de Ugarte Postigo et al. 2005).

The millimeter detection 3 days after the burst and an upper limit around 5 days after the burst (see Table 1) puts an important constraint on the temporal decay of the afterglow. Comparison of the millimeter and optical flux at the similar epochs in terms of a simple ISM jet model shows an order of $\sim 2$ mag excess emission at millimeter frequencies with respect to predictions. The expected millimeter flux was calculated using the value of the maximum synchrotron frequency $v_{\mathrm{m}}$ at millimeter frequencies at the epoch of the observations, the self absorption break frequency $v_{a}$ at radio frequencies, $v_{\mathrm{c}}$ above the X-ray band, and the derived values of the jet-break time and $p$ were as discussed above. The derived excess may imply the presence of variability in the millimeter regime and possibly that energy injection contributes significantly in this band. The possibility of a millimeter bright host galaxy at this high redshift is ruled out on the basis of upper limits obtained from our subsequent monitoring of the field around 160 days after the burst.

The gamma-ray fluence of $4.4 \times 10^{-6} \mathrm{erg} / \mathrm{cm}^{2}$ in the energy band 15-350 keV with the measured redshift $z=3.967 \pm$ 0.001 implies an isotropic equivalent energy release $E_{\text {iso }, \gamma} \sim$ $2.3 \times 10^{53}$ erg assuming $H_{0}=65 \mathrm{~km} \mathrm{~s}^{-1} \mathrm{Mpc}^{-1}$ in a $\Omega_{0}=0.3$ and $\Lambda_{0}=0.7$ cosmological model taking into account the cosmological K-correction (Bloom et al. 2001). If we take the observed break time of 0.1 days as the jet-break time and an assumed value of $\gamma$-ray efficiency $\eta_{\gamma}=0.2$, this leads to an estimated jet half-opening angle $\theta \sim 1.3 \times n^{1 / 8}$ degrees, where $n$ is the particle density of the ambient media. The total $\gamma$-ray energy output in the jet is $E_{\gamma} \sim 5.7 \times 10^{49}$ erg which lies at the low end of the observed beaming corrected values of $E_{\gamma}$ for long duration GRBs (Frail et al. 2001) and appears to be an underluminous burst. A possible explanation for the below average value of $E_{\gamma}$ may be either considerable energy deposited at lower frequencies in form of energy injection episodes or in afterglow kinetic energy. The observed early jet-break time implies that this burst was viewed almost pole-on. Although the multi-wavelength observations of GRB 050730 have uncovered the peculiar nature of the afterglow, the gaps in the light-curves make it impossible to distinguish between several possible scenarios.

\section{Conclusion}

Multi-wavelength observations of the afterglow of GRB 050730 from millimeter to NIR and optical frequencies are used to analyze the burst properties. The unusual nature of the 
superimposed variability is seen in the light-curves from millimeter to X-rays. The derived values of the photon indices from the Swift and XMM-Newton data analysis along with the optical and NIR $\beta$ constrain the value of $p \sim 2.3$ and the location of the cooling break to be above the observed frequencies. Model predicted flux at millimeter frequencies shows an excess in the observed millimeter fluxes around 3 days after the burst, indicating possible variability. The value of $\alpha_{1}$ derived from optical observations suggests early time energy injections. The derived value of the jet-break time shows the burst to be under-luminous on the basis of the derived beaming corrected value of $E_{\gamma}$. Detailed modeling is encouraged to understand the nature of the observed variability and the missing energy in the form of possible energy injection episodes in this case.

The importance of early time multi-wavelength NIRandoptical observations is obvious in the case of the high redshift burst GRB 050730. The observed early time ( $\sim 0.1$ day in the observer's frame) break in the optical light-curves of this high redshift event, interpreted in terms of a jet-break, is not common in comparison with other well observed afterglows at lower redshifts. In future, X-ray afterglow observations at late phases will be essential in order to understand these primordial energetic explosions.

Acknowledgements. This research has made use of data obtained through the High Energy Astrophysics Science Archive Research Center On line Service, provided by the NASA/Goddard Space Flight Center. S.B.P. acknowledges MAE-AECI grant for this work. J.G. and J.A.C. are researchers of the programme Ramón y Cajal funded jointly by the Spanish Ministerio de Educación y Ciencia (former Ministerio de Ciencia y Tecnología) and Universidad de Jaén. The authors also acknowledge support by DGI of the Spanish Ministerio de Educación y Ciencia under grants AYA2004-07171-C02-02, FEDER funds and Plan Andaluz de Investigación of Junta de Andalucía as research group FQM322. M.A.G. acknowledges support by the Spanish program Ramón y Cajal. SMB acknowledges the support of the European Union through a Marie Curie IntraEuropean Fellowship within the Sixth Framework Program. Millimeter observations from IRAM Plateau de Bure Interferometer are acknowledged. IRAM is supported by INSU/CNRS (France), MPG (Germany) and ING (Spain). The Liverpool Telescope is operated on the island of La Palma by Liverpool John Moores University at the Observatorio del Roque de los Muchachos of the Instituto de Astrofísica de Canarias. Publicly available Swift/XRT and UVOT data and Leicester University HelpDesk are also acknowledged. We are also thankful to the anonymous referee for the constructive comments which significantly improved the paper.

\section{References}

Arnaud, K. A. 1996, ASP Conf. Ser., 101, 17

Bessell, M. S., Castelli, F., \& Plez, B. 1998, A\&A, 333, 231

Beuermann, K., Hessman, F. V., Reinsch, K., et al. 1999, A\&A, 352, L26

Bhatt, B. C., \& Sahu, D. K. 2005, GCN Circ., 3775
Björnsson, G., Hjorth, J., Pedersen, K., \& Fynbo, J. P. U. 2002, ApJ, 579, L59 Bloom, J. S., Frail, D. A., \& Sari, R. 2001, AJ, 121, 2879

Blustin, A. J., Holland, S. T., Cucchiara, A., et al. 2005, GCN Circ., 3717

Boër, M., Atteia, J. L., Damerdji, Y., et al. 2006, ApJ, 638, L71

Burenin, R., Tkachenko, A., Pavlinsky, M., et al., 2005, GCN Circ., 3718

Burrows, D. N., Romano, P., Falcone, A., et al. 2005, Science, 309, 1833

Cameron, P. B. 2005, GCN Circ., 3761

Chen, H. W., Thompson, I. B., Prochaska, J. X., \& Bloom, J. S. 2005a, GCN Circ., 3709

Chen, H. W., Prochaska, J. X., Bloom, J. S., \& Thompson, I. B. 2005b, ApJ, 634, L25

Cobb, B. E., \& Bailyn, C. D. 2005, GCN Circ., 3708

Cusumano, G., Mangano, V., Angelini, L., et al. 2006, ApJ, 639, 316

Cusumano, G., Mangano, V., Chincarini, G., et al. 2006, Nature, 440, 164

Damerdji, Y., Klotz, A., Boer, M., \& Atteia, J. L. 2005, GCN Circ., 3741

de Ugarte Postigo, A., Castro-Tirado, A. J., Gorosabel, J., et al. 2005, A\&A, 443, 841

Dickey, J. M., \& Lockman, F. J. 1990, ARA\&A, 28, 215

Ehle, M., \& Juarez, B. 2005, GCN Circ., 3713

Frail, D. A., Kulkarni, S. R., Sari, R., et al. 2001, ApJ, 562, L55

Frontera, F., Amati, L., Lazzati, D., et al. 2004, ApJ, 614, 301

Gehrels, N., Chincarini, G., Giommi, P., et al. 2004, ApJ, 611, 1005

Gomboc, A., Guidorzi, C., Steele, I. A., et al. 2005, GCN Circ., 3706

Grupe, D., Kennea, J. A., \& Burrows, D. N. 2005, GCN Circ., 3714

Guilloteau, S., Delannoy, J., Downes, D., et al. 1992, A\&A, 262, 624

Haislip, J., Kirschbrown, J., Reichart, D., et al. 2005, GCN Circ., 3712

Holland, S. T., Barthelmy, S., Burrows, D. N., et al. 2005, GCN Circ., 3704

Kannappan, S., Garnavich, P., Stanek, K. Z., et al. 2005, GCN Circ., 3778

King, A., O’Brien, P., Goad, M. R., et al. 2005, ApJ, 630, L113

Landolt, A. R. 1992, AJ, 104, 340

Lazzati, D., Rossi, E., Covino, S., Ghisellini, G., \& Malesani, D. 2002, A\&A, 396, L5

Markwardt, C. B., Barbier, L., Barthelmy, S., et al. 2005, GCN Circ., 3715

Mathis, J. S. 1990, ARA\&A, 28, 37

Nakar, E., Piran, T., \& Granot, J. 2003, New Astr., 8, 495

Pandey, S. B., Sahu, D. K., Resmi, L., et al. 2003, Bull. Astro. Soc. India, 31, 19

Perna, R., Armitage, P. J., \& Zhang, B. 2006, ApJ, 636, L29

Perri, M., et al. 2005, GCN Circ., 3722

Persson, S. E., Murphy, D. C., Krzeminski, W., et al. 1998, AJ, 116, 247

Prochaska, J. X., Chen, H. W., Bloom, J. S., et al. 2005, GCN Circ., 3732

Proga, D., \& Zhang, B. 2006, ApJ, submitted [arXiv: astro-ph/0601272]

Rol, E., Starling, R., Wiersema, K., et al. 2005, GCN Circ., 3710

Rhoads, J. 1999, ApJ, 487, L1

Sagar, R., Mohan, V., Pandey, S. B., et al. 2000, Bull. Astro. Soc. India, 28, 499

Sari, R., Piran, T., \& Narayan, R. 1998, ApJ, 497, L17

Sari, R., Piran, T., \& Halpern 1999, ApJ, 519, L17

Schartel, N. 2005, GCN Circ., 3707

Schlegel, D. J., Finkbeiner, D. P., \& Davis, M. 1998, ApJ, 500, 525

Sota, A., Castro-Tirado, A. J., Guziy, S., et al. 2005, GCN Circ., 3705

Starling, R. L. C., Vreeswijk, P. M., Ellison, S. L., et al. 2005, A\&A, 442, L21

Tagliaferri, G., Antonelli, L. A., Chincarini, G., et al. 2005, A\&A, 442, L21

van der Horst, A. J., \& Rol, E. 2005a, GCN Circ., 3781

van der Horst, A. J., \& Rol, E. 2005b, GCN Circ., 3810

Vaughan, S., Goad, M. R., Beardmore, A. P., et al. 2006, Ap.J. 638, 920-929

Watson, D., Reeves, J. N., Hjorth, J., et al. 2006, ApJ, 637, L69

Zhang, B., \& Mészáros, P. 2002, ApJ, 566, 712 